\title{
A Weak Coherence Theorem and Remarks to the Oka Theory
}

\author{
By Junjiro Noguchi*
}

\begin{abstract}
The proofs of K. Oka's Coherence Theorems are based on Weierstrass' Preparation (division) Theorem. Here we formulate and prove a Weak Coherence Theorem without using Weierstrass' Preparation Theorem, but only with power series expansions: The proof is almost of linear algebra. Nevertheless, this simple Weak Coherence Theorem suffices to give other proofs of the Approximation, Cousin I/II, and Levi's (Hartogs' Inverse) Problems even in simpler ways than those known, as far as the domains are non-singular; they constitute the main basic part of the theory of several complex variables.

The new approach enables us to complete the proofs of those problems in quite an elementary way without Weierstrass' Preparation Theorem or the cohomology theory of Cartan-Serre, nor $L^{2}-\bar{\partial}$ method of Hörmander.

We will also recall some new historical facts that Levi's (Hartogs' Inverse) Problem of general dimension $n \geq 2$ was, in fact, solved by K. Oka in 1943 (unpublished) and by S. Hitotsumatsu in 1949 (published in Japanese), whereas it has been usually recognized as proved by K. Oka 1953, by H.J. Bremermann and by F. Norguet 1954, independently.
\end{abstract}

\section{Introduction and a weak coherence}

K. Oka [28], 29] proved three fundamental coherence theorems for

(i) the sheaf $\mathcal{O}:=\mathcal{O}_{\mathbf{C}^{n}}$ of germs of holomorphic functions on $\mathbf{C}^{n}$,

(ii) the ideal sheaf $\mathscr{I}\langle A\rangle$ of an analytic subset $A$ of an open subset of $\mathbf{C}^{n}$,

(iii) the normalization of the structure sheaf of a complex space,

where for the second, H. Cartan [4] gave his own proof based on Oka 28] (cf. 17] Chap. 9). We simply call $\mathscr{I}\langle A\rangle$ a (resp. non-singular) geometric ideal sheaf of a (resp. non-singular) analytic subset $A$ (cf., e.g., 17] Chap. 6). Oka's Coherence has played a fundamental role in modern Mathematics, so that it led to the notion of ringed spaces due to H. Cartan, and developed by J.-P. Serre, R. Remmert, H. Grauert and A. Grothendieck (cf. [6] p. 162). The proofs of the above coherence theorems rely on Weierstrass' Preparation (division) Theorem.

The aim of this paper is to formulate a Weak Coherence Theorem (Theorem 1.2 below), which we prove not with Weierstrass' Preparation Theorem, but only with power series expansions, and then to apply it to prove the Approximation Problem, Cousin I/II Problems, $\bar{\partial}$-equation (for functions), holomorphic

\footnotetext{
*Research supported in part by Grant-in-Aid for Scientific Research (C) 15K04917.

AMC2010: 32A99; 32E30

Key words: coherence, Oka, Levi problem, Hartogs' inverse problem, several complex variables

Affiliation and address: Graduate School of Mathematical Sciences, University of Tokyo (Emeritus); Komaba, Meguroku, Tokyo 153-8914, Japan

e-mail: noguchi@ms.u-tokyo.ac.jp
} 
extensions (interpolations), and Levi's (Hartogs' Inverse) Problem for unramified Riemann domains (over $\mathbf{C}^{n}$ (see Theorem 4.11 and 4.3 ; t they constitute the main basic part of the theory of several complex variables. The proofs are even simpler than those in the standard references (cf., e.g., Gunning-Rossi [10], Grauert-Remmert 9], Hörmander [12, Noguchi [17]).

Note that the present approach enables us to complete the proofs of those problems in quite an elementary way without Weierstrass' Preparation Theorem or the cohomology theory $\left.{ }^{2}\right)$ of Cartan-Serre, nor $L^{2}-\bar{\partial}$ method of Hörmander. The present paper came out from the study of the degree structure of a generator system of a coherent analytic sheaf by [16.

Let $\Omega$ denote a domain of $\mathbf{C}^{n}$ with the structure sheaf $\mathcal{O}=\mathcal{O}_{\Omega}$. For a holomorphic function $f \in \mathcal{O}(\Omega)$ in $\Omega$ we write $\underline{f} \in \Gamma(\Omega, \mathcal{O})$ for the induced sheaf-section of $\mathcal{O}$ and $\underline{f}_{z}$ for the germ of $f$ at $z \in \Omega$. Let $\mathscr{F}$ be an analytic sheaf on $\Omega$ (i.e., a sheaf of $\mathcal{O}$-modules over $\Omega$ ), and let $\xi_{j} \in \Gamma(\Omega, \mathscr{F}), 1 \leq j \leq q$, be finitely many sections on $\Omega$. Then the relation sheaf $\mathscr{R}\left(\xi_{1}, \ldots, \xi_{q}\right)$ of $\left\{\xi_{j}\right\}_{j=1}^{q}$ is a subsheaf of $\mathcal{O}^{q}$ consisting of those germ-vectors $\left(\underline{f}_{z}, \ldots, \underline{f}_{z}\right) \in \mathcal{O}_{z}^{q}$ such that

$$
\underline{f}_{z} \xi_{1}(z)+\cdots+\underline{f}_{z} \xi_{q}(z)=0, \quad z \in \Omega .
$$

Now we formulate:

Theorem 1.2 (Weak Coherence). Let $S \subset \Omega$ be a complex submanifold ${ }^{3)}$

(i) The non-singular geometric ideal sheaf $\mathscr{I}\langle S\rangle$ is locally finite.

(ii) Let $\left\{\underline{\sigma_{j}} \in \Gamma(\Omega, \mathscr{I}\langle S\rangle): 1 \leq j \leq N\right\}$ be a finite generator system of $\mathscr{I}\langle S\rangle$ on $\Omega$ with $\sigma_{j} \in \mathcal{O}(\Omega)$ : i.e.,

$$
\mathscr{I}\langle S\rangle=\sum_{j=1}^{N} \mathcal{O} \cdot \underline{\sigma_{j}} .
$$

Then, the relation sheaf $\mathscr{R}\left(\underline{\sigma_{1}}, \ldots, \underline{\sigma_{N}}\right)$ is locally finite.

We give a proof of this theorem in $\S 2$. In $\S 3$ we will apply it to prove Oka's Jôku-Ik $\hat{q}^{4}$ (see Lemma 3.11), and then we will give a unified proof for Cousin I/II Problems, and $\bar{\partial}$-equation for functions in $\S 4$ (Theorem 4.11) by combining the Weak Coherence Theorem 1.2 with a method of cuboid induction on dimension; these yield $H^{1}(\Omega, \mathcal{O})=0$ for a holomorphically convex domain $\Omega$ (Lemma 4.20 , which suffices to derive Oka's Heftungslemma or Grauert's finiteness theorem for $\mathcal{O}$ on a strongly pseudoconvex domain (Theorem 4.21). In 4.3 we finally give the solution of Levi's (Hartogs' Inverse) Problem for unramified Riemann domains.

\section{Proof of Theorem 1.2}

(i) We take an arbitrary point $a \in \Omega$.

\footnotetext{
1) In the present, "over $\mathbf{C}^{n}$ " will be abbreviated, unless necessary.

2) We use only the 1-cocyle class space $H^{1}(*, \mathcal{O})$ as a complex vector space.

3) A complex submanifold is not necessarily connected in this paper.

4) This is a method or a principle of K. Oka all through his series of papers 20 - 30 such that to solve a problem on a difficult domain one embeds the domain into a higher dimensional polydisk, extends the problem on the polydisk, and then solves it by making use of the simple shape of the polydisk (cf. [17]).
} 
Case of $a \notin S$ : Since $S$ is closed, there is a neighborhood $U \subset \Omega$ of $a$ with $U \cap S=\emptyset$. Then,

$$
\mathscr{I}\langle S\rangle_{x}=\mathcal{O}_{x}=1 \cdot \mathcal{O}_{x}, \quad{ }^{\forall} x \in U
$$

and therefore, $\{1\}$ is a finite generator system of $\mathscr{I}\langle S\rangle_{x}$ on $U$.

Case of $a \in S$ : There is a holomorphic local coordinate neighborhood $U$ of $a$ with $z=\left(z_{1}, \ldots, z_{n}\right)$ such that

$$
\begin{aligned}
a & =(0, \ldots, 0) \in U=\mathrm{P} \Delta\left(0 ;\left(r_{j}\right)\right), \\
S \cap U & =\left\{z=\left(z_{j}\right) \in U: z_{1}=\cdots=z_{q}=0\right\} \quad\left(1 \leq{ }^{\exists} q \leq n\right),
\end{aligned}
$$

where $\mathrm{P} \Delta\left(0 ;\left(r_{j}\right)\right)=\left\{\left(z_{j}\right) \in \mathbf{C}^{n}:\left|z_{j}\right|<r_{j}, 1 \leq j \leq n\right\}$ is a polydisk with center at 0 . Let $\underline{f}_{b} \in \mathscr{I}\langle S\rangle_{b}$ $(b \in U \cap S)$ be any element. With the coordinate system $\left(z_{j}\right)$ we write $b=\left(b_{j}\right)=\left(0, \ldots, 0, b_{q+1}, \ldots, b_{n}\right)$. The function $f$ is represented by a unique power series expansion, $f(z)=\sum_{\nu \in \mathbf{Z}_{+}^{n}} c_{\nu}(z-b)^{\nu}$, which decomposes to

$$
\begin{aligned}
f(z) & =\sum_{\nu=\left(\nu_{1}, \nu^{\prime}\right) \in \mathbf{Z}_{+}^{n}, \nu_{1}>0} c_{\nu}(z-b)^{\nu}+\sum_{\nu=\left(\nu_{1}, \nu^{\prime}\right) \in \mathbf{Z}_{+}^{n}, \nu_{1}=0} c_{\nu}(z-b)^{\nu} \\
& =\left(\sum_{\nu=\left(\nu_{1}, \nu^{\prime}\right) \in \mathbf{Z}_{+}^{n}, \nu_{1}>0} c_{\nu} z_{1}^{\nu_{1}-1}\left(z^{\prime}-b^{\prime}\right)^{\nu^{\prime}}\right) z_{1}+\sum_{\nu^{\prime} \in \mathbf{Z}_{+}^{n-1}} c_{0 \nu^{\prime}}\left(z^{\prime}-b^{\prime}\right)^{\nu^{\prime}} .
\end{aligned}
$$

Here we put $\nu^{\prime}=\left(\nu_{2}, \ldots, \nu_{n}\right), z^{\prime}=\left(z_{2}, \ldots, z_{n}\right)$, and $b^{\prime}=\left(b_{2}, \ldots, b_{n}\right)$. Setting

$$
\begin{aligned}
h_{1}\left(z_{1}, z^{\prime}\right) & =\left(\sum_{\nu=\left(\nu_{1}, \nu^{\prime}\right) \in \mathbf{Z}_{+}^{n}, \nu_{1}>0} c_{\nu} z_{1}^{\nu_{1}-1}\left(z^{\prime}-b^{\prime}\right)^{\nu^{\prime}}\right), \\
g_{1}\left(z^{\prime}\right) & =\sum_{\nu^{\prime} \in \mathbf{Z}_{+}^{n-1}} c_{0 \nu^{\prime}}\left(z^{\prime}-b^{\prime}\right)^{\nu^{\prime}},
\end{aligned}
$$

we have

$$
f\left(z_{1}, z^{\prime}\right)=h_{1}\left(z_{1}, z^{\prime}\right) \cdot z_{1}+g_{1}\left(z^{\prime}\right)
$$

For $g_{1}\left(z^{\prime}\right)$ we apply a similar decomposition with respect to variable $z_{2}$, so that

$$
g_{1}\left(z^{\prime}\right)=h_{2} \cdot z_{2}+g_{2}\left(z^{\prime \prime}\right), \quad z^{\prime \prime}=\left(z_{3}, \ldots, z_{n}\right)
$$

Repeating this process, we get

$$
f(z)=\sum_{j=1}^{q} h_{j}(z) \cdot z_{j}+g_{q}\left(z_{q+1}, \ldots, z_{n}\right) .
$$

If $z_{1}=\cdots=z_{q}=0$, then $f(z)=0$, and so $g_{q}\left(z_{q+1}, \ldots, z_{n}\right)=0$. Therefore,

$$
f(z)=\sum_{j=1}^{q} h_{j}(z) \cdot z_{j}
$$


Thus,

$$
\left.\mathscr{I}\langle S\rangle\right|_{U}=\sum_{j=1}^{q} \mathcal{O}_{U} \cdot \underline{z_{j}} .
$$

(ii) We begin with the following lemma:

Lemma 2.4. With the natural complex coordinate system $z=\left(z_{1}, \ldots, z_{n}\right) \in \mathbf{C}^{n}$ we consider a relation sheaf $\mathscr{R}_{p}(1 \leq p \leq n)$ defined by

$$
\underline{f_{1}} z \underline{z_{1}} z+\cdots+\underline{f_{p}} z \underline{z_{p}} z=0, \quad \underline{f_{j}} \in \mathcal{O}_{z} .
$$

Then $\mathscr{R}_{p}$ is finitely generated on $\mathbf{C}^{n}$ by

$$
T_{i j}=\left(0, \ldots, 0, \stackrel{i \text {-th }}{z_{j}}, 0, \ldots, 0, \stackrel{j \text {-th }}{z_{i}}, 0, \ldots, 0\right), \quad 1 \leq i<j \leq p .
$$

We call $T_{i j}(1 \leq i<j \leq p)$ of 2.6 the trivial solutions of 2.5 or of $\mathscr{R}_{p}$. In the case of $p=1$, we set the trivial solution to be 0 as a convention.

Proof of Lemma 2.4: We use induction on $p \geq 1$. The case of $p=1$ is clear.

Assuming that the case of $p-1(p \geq 2)$ holds, we consider the case of $p$. Set

$$
\Sigma=\left\{\left(z_{1}, \ldots, z_{n}\right): z_{1}=\cdots=z_{p}=0\right\},
$$

and let $a \in \mathbf{C}^{n}$ be an arbitrary point. If $a=\left(a_{j}\right) \notin \Sigma$, there is an $a_{j} \neq 0(1 \leq j \leq p)$, to say, $a_{1} \neq 0$. In a neighborhood $V$ of $a, z_{1} \neq 0$. Then, 2.5 is solvable with respect to $\underline{f_{1}}$ :

$$
\underline{f_{1}} z=-\underline{f_{2}} z \cdot \frac{\underline{z}_{z}}{\underline{z}_{z}}-\cdots-\underline{f_{p}} \cdot \frac{\underline{z}_{z}}{\underline{\underline{z_{1}}}}, \quad{ }^{\forall} \underline{f}_{j} \in \mathcal{O}_{z}(2 \leq j \leq p), \quad z \in V .
$$

It follows that with $z \in V$,

$$
\begin{aligned}
\left(\underline{f_{j}}\right) & =\left(-\sum_{j=2}^{p} \underline{f_{j}} z \cdot \frac{\underline{z}_{j}}{\underline{z_{1}}}, \underline{f_{2}}, \ldots, \underline{f_{p}} z\right) \\
& =\sum_{j=2}^{p} \frac{\underline{f_{j}}}{\overline{z_{1}} z} \cdot\left(-\underline{z_{j}} z, 0, \ldots, 0, \underline{z_{1}} z, 0, \ldots, 0\right) \\
& =\sum_{j=2}^{p}-\frac{f_{j}}{\underline{z_{1}} z} \cdot T_{1 j}(z) \in \sum_{j=2}^{p} \mathcal{O}_{z} \cdot T_{1 j}(z) .
\end{aligned}
$$

Therefore, $\mathscr{R}_{p}$ is generated by the trivial solutions $\left\{T_{1 j}\right\}_{2 \leq j \leq p}$ on $V$.

If $a \in \Sigma$, we decompose an element $\left(\underline{f}_{j}\right) \in \mathscr{R}_{p a}$ in a polydisk neighborhood $U$ of $a$ as in 2.2):

$$
f_{j}\left(z_{1}, z^{\prime}\right)=h_{j}\left(z_{1}, z^{\prime}\right) z_{1}+g_{j}\left(z^{\prime}\right), \quad z^{\prime}=\left(z_{2}, \ldots, z_{n}\right), 1 \leq j \leq p .
$$

For $z \in U$ one gets

$$
\begin{aligned}
\left(\underline{f_{j}}\right)-\sum_{j=2}^{p} \underline{h_{j}} T_{1 j}(z) & =\left(\underline{g_{1}} z+\sum_{j=1}^{p} \underline{h_{j}} z \underline{z_{j}}, \underline{g_{2}}, \ldots, \underline{g_{p}} z\right) \\
& =\left(\underline{\tilde{g}}_{z}, \underline{g_{2}}, \ldots, \underline{g_{p}}\right) .
\end{aligned}
$$


Here, $\underline{\tilde{g}}_{z}=\underline{g}_{z}+\sum_{j=1}^{p} \underline{h}_{z} \underline{z}_{z}$. Since $\left(\underline{\tilde{g}}_{z}, \underline{g_{2}}, \ldots, \underline{g_{p}} z\right) \in \mathscr{R}_{p_{z}}$,

$$
\underline{\tilde{g}_{1}} z \underline{z}_{z}+\underline{g_{2}} z \underline{z_{2}} z+\cdots+\underline{g_{p}} z \underline{z_{p}} z=0 .
$$

The second term and so forth of the right-hand side of the equation above do not contain variable $z_{1}$, and so $\underline{\tilde{g}}_{z}=0$ is deduced. Thus,

$$
\underline{g_{2}} z \underline{z_{2}} z+\cdots+\underline{g_{p}} z \underline{z_{p}} z=0
$$

This is the case of $p-1$ after changing the indices of variables. Therefore, the induction hypothesis implies that $\left(0, \underline{g}_{z}, \ldots, \underline{g}_{z}\right)$ is represented as a linear sum of $T_{i j}(z), 2 \leq i<j \leq p$, with coefficients in $\mathcal{O}_{z}$. Combining this with 2.8 , we see that $\left(\underline{f}_{z}\right)$ is represented as a linear sum of $T_{i j}(z), 1 \leq i<j \leq p$, with coefficients in $\mathcal{O}_{z}$.

Continued proof of (ii): Set $\mathscr{R}=\mathscr{R}\left(\underline{\sigma_{1}}, \ldots, \underline{\sigma_{N}}\right)$. We consider the relation

$$
\underline{f_{1}} z{\underline{\sigma_{1}}}_{z}+\cdots+\underline{f}_{N_{z}}{\underline{\sigma_{N}}}_{z}=0, \quad \underline{f}_{z} \in \mathcal{O}_{z} .
$$

We set the trivial solutions of this equation as follows:

$$
\tau_{i j}=\left(\ldots, \stackrel{i-\mathrm{th}_{j}}{-\sigma_{j}}, \ldots, \underline{j-\text { th }}, \ldots\right), \quad 1 \leq i<j \leq N .
$$

We take an arbitrary point $a \in \Omega$. If $a \notin S$, then some $\sigma_{j}(a) \neq 0$, to say, $\sigma_{1}(a) \neq 0$. As in (2.7), one sees that $\mathscr{R}$ is generated by $\left\{\tau_{1 j}\right\}_{j=2}^{N}$ on a neighborhood of $a$.

If $a \in S$, we take a holomorphic local coordinate system $z=\left(z_{1}, \ldots, z_{n}\right)$ in a polydisk neighborhood $\mathrm{P} \Delta$ as in 2.1):

$$
\begin{aligned}
a & =(0, \ldots, 0), \\
S \cap \mathrm{P} \Delta & =\left\{\left(z_{1}, \ldots, z_{n}\right) \in \mathrm{P} \Delta: z_{1}=\cdots=z_{q}=0\right\} \quad\left(1 \leq{ }^{\exists} q \leq n\right) .
\end{aligned}
$$

It follows from (2.3) and the assumption that

$$
\left.\mathscr{I}\langle S\rangle\right|_{\mathrm{P} \Delta}=\sum_{j=1}^{q} \mathcal{O}_{\mathrm{P} \Delta} \cdot \underline{z_{j}}=\left.\sum_{j=1}^{N} \mathcal{O}_{\mathrm{P} \Delta} \cdot \underline{\sigma_{j}}\right|_{\mathrm{P} \Delta}
$$

Thus, we may assume without loss of generality that

$$
\begin{aligned}
\sigma_{j} & =z_{j}, \quad 1 \leq j \leq q(\text { on } \mathrm{P} \Delta) \\
\sigma_{i} & =\sum_{j=1}^{q} a_{i j} z_{j}, \quad a_{i j} \in \mathcal{O}(\mathrm{P} \Delta), q+1 \leq i \leq N(\text { on } \mathrm{P} \Delta) .
\end{aligned}
$$

Set

$$
\phi_{i}=\left(-\underline{a_{i 1}}, \ldots,-\underline{a_{i q}}, 0, \ldots, 0, \stackrel{i \text { th }}{1}, 0, \ldots, 0\right) \in \Gamma(\mathrm{P} \Delta, \mathscr{R}), \quad q+1 \leq i \leq N .
$$

We deduce from 2.9 with $z \in \mathrm{P} \Delta$ that

$$
\left(\underline{f}_{z}+\sum_{i=q+1}^{N} \underline{f}_{z} \underline{a_{i 1}} z\right) \underline{z}_{z}+\cdots+\left(\underline{f_{q}} z+\sum_{i=q+1}^{N} \underline{f_{i}} z \underline{a_{i q}} z\right) \underline{z}_{z}=0 .
$$


By Lemma 2.4 .

$$
\left(\underline{f}_{z}+\sum_{i=q+1}^{N} \underline{f}_{z} \underline{a_{i 1}} z, \ldots, \underline{f_{q}} z+\sum_{i=q+1}^{N} \underline{f_{i}} z \underline{a_{i q}} z, 0, \ldots, 0\right)
$$

is a linear sum of $\tau_{j k}(z), 1 \leq j<k \leq q$, with coefficients in $\mathcal{O}_{z}$. Therefore there are $\underline{b_{j k}} \in \mathcal{O}_{z}$, $1 \leq j<k \leq q$, such that

$$
\begin{aligned}
\sum_{1 \leq j<k \leq q} \underline{b_{j k}} z \tau_{j k}(z) & =\left(\underline{f_{1}} z+\sum_{i=q+1}^{N} \underline{f_{i}} z \underline{a_{i 1}} z, \ldots, \underline{f_{q}} z+\sum_{i=q+1}^{N} \underline{f_{i}} z \underline{a_{i q}} z, 0, \ldots, 0\right) \\
& =\left(\underline{f_{1}} z, \ldots, \underline{f_{q}} z, 0, \ldots, 0\right)+\sum_{i=q+1}^{N} \underline{f_{i}} z\left(\underline{a_{i 1}} z, \ldots, \underline{a_{i q}} z, 0, \ldots, 0\right) .
\end{aligned}
$$

By making use of 2.10 we get

$$
\left(\underline{f}_{z}, \ldots, \underline{f_{q}}, \ldots, \underline{f_{N}}\right)=\sum_{1 \leq j<k \leq q} \underline{b_{j k}} z \tau_{j k}(z)+\sum_{i=q+1}^{N} \underline{f}_{z} \phi_{i}(z) .
$$

Thus, $\mathscr{R}$ is generated on $\mathrm{P} \Delta$ by

$$
\tau_{j k}, \phi_{i}, \quad 1 \leq j<k \leq q, q+1 \leq i \leq N .
$$

This finishes the proof.

Remark 2.15. (i) In the Weak Coherence Theorem 1.2 it is a point to assume that $\left\{\sigma_{j}\right\}_{j=1}^{N}$ is a generator system of $\mathscr{I}\langle S\rangle$; otherwise, the proof above does not work even if $S$ is non-singular.

(ii) It is an advantage of the above method to the general First Coherence Theorem of Oka that we have an explicit system of generators 2.14.

\section{Oka's Jôku-Ikô}

The term "Jôku-Ikô" was used by K. Oka since he wrote the first paper of the series in 1936 and retained this principle all through his works $([20]-30]$ ) (see footnote 4)]; The aim of the present section is to prove Oka's Jôku-Ikô, Lemma 3.11 below only by making use of Theorem 1.2 combined with Cousin's integral (3.7). The technics may be essentially similar to those in some references, e.g., Nishino [14] and Noguchi [17, but they are not in a suitable form for our purpose.

\subsection{Syzygy for non-singular geometric ideal sheaves}

We begin with:

Definition 3.1. A cuboid $E$ is a bounded open or closed subset of $\mathbf{C}^{n}$ with the boundary parallel to the real and imaginary axes of $z=\left(z_{1}, \ldots, z_{n}\right) \in \mathbf{C}^{n}$. In the case of $n=1, E$ is called a rectangle. When $E$ is a closed cuboid, we allow the widths of some edges to degenerate to 0 , and call the number of edges of $E$ of positive widths the dimension of $E$, denoted by $\operatorname{dim} E$. 
Let $\Omega \subset \mathbf{C}^{n}=\mathbf{C}^{n-1} \times \mathbf{C}$ be a domain and let $E^{\prime}, E^{\prime \prime} \Subset \Omega^{5)}$ be two closed cuboids as follows: There are a closed cuboid $F \Subset \mathbf{C}^{n-1}$ and two adjacent closed rectangles $E_{n}^{\prime}, E_{n}^{\prime \prime} \Subset \mathbf{C}$ sharing a side $\ell$, and

$$
E^{\prime}=F \times E_{n}^{\prime}, \quad E^{\prime \prime}=F \times E_{n}^{\prime \prime}, \quad \ell=E_{n}^{\prime} \cap E_{n}^{\prime \prime} .
$$

Fig_4_2tt_2.pdf

Figure 1: Adjacent closed cuboids

We now recall:

Lemma 3.3 (Cartan's Merging Lemma). Let $E^{\prime}, E^{\prime \prime} \Subset \Omega$ be adjacent closed cuboids as in (3.2), and let $\mathscr{F}$ be an analytic sheaf on $\Omega$. Let $\left\{\sigma_{j}^{\prime} \in \Gamma\left(U^{\prime}, \mathscr{F}\right): 1 \leq j \leq p^{\prime}\right\} \quad\left(\right.$ resp. $\left.\left\{\sigma_{k}^{\prime \prime} \in \Gamma\left(U^{\prime \prime}, \mathscr{F}\right), 1 \leq k \leq p^{\prime \prime}\right\}\right)$ be a finite generator system of $\mathscr{F}$ on $E^{\prime}$ (resp. $\left.E^{\prime \prime}\right){ }^{6}$

Moreover, assume that there are holomorphic functions $a_{j k}, b_{k j} \in \mathcal{O}\left(E^{\prime} \cap E^{\prime \prime}\right), 1 \leq j \leq p^{\prime}, 1 \leq k \leq p^{\prime \prime}$, such that

$$
\sigma_{j}^{\prime}=\sum_{k=1}^{p^{\prime \prime}} \underline{a_{j k}} \cdot \sigma_{k}^{\prime \prime}, \quad \sigma_{k}^{\prime \prime}=\sum_{j=1}^{p^{\prime}} \underline{b_{k j}} \cdot \sigma_{j}^{\prime} \quad\left(\text { on } E^{\prime} \cap E^{\prime \prime}\right) .
$$

Then, there exists a merged finite generator system $\left\{\sigma_{l} \in \Gamma\left(E^{\prime} \cup E^{\prime \prime}, \mathscr{F}\right): 1 \leq l \leq p^{\prime}+p^{\prime \prime}\right\}$ on $E^{\prime} \cup E^{\prime \prime}$.

This is due to H. Cartan 1940; a rather simplified proof of it can be found in [17, "Added at galleyproof".

Lemma 3.4 (Oka's Syzygy). Let $E \Subset \mathbf{C}^{n}$ be a closed cuboid.

(i) Every locally finite analytic sheaf $\mathscr{F}$ defined on $E$ (i.e., in a neighborhood of $E$ ) has a finite generator system on $E$.

(ii) Let $\mathscr{F}$ be an analytic sheaf on $E$ with a finite generator system $\left\{\sigma_{j}\right\}_{1 \leq j \leq N}$ on $E$ such that the relation sheaf $\mathscr{R}\left(\sigma_{1}, \ldots, \sigma_{N}\right)$ is locally finite.

Then for every section $\sigma \in \Gamma(E, \mathscr{F})$ there are holomorphic functions $a_{j} \in \mathcal{O}(E), 1 \leq j \leq N$, such that

$$
\sigma=\sum_{j=1}^{N} \underline{a_{j}} \cdot \sigma_{j} \quad(\text { on } E) .
$$

Proof. The proof is carried out in the same way as in [14, or [17] Lemma 4.3.7 except for the use of the vanishing $H^{1}(U, \mathcal{O})=0$ for an affine convex cylinder domain $U \subset \mathbf{C}^{n}$, which we replace by Cousin's

5) The symbol "๔" stands for that the inclusion is relatively compact.

6) This means that they are defined so in some neighborhoods of $E^{\prime}$ and $E^{\prime \prime}$, respectively; this expression is the same through the paper. 
integral (3.7) as follows. Suppose that $E$ is a closed cuboid such that

$$
E=F \times\left\{z_{n}:\left|\Re z_{n}\right| \leq T,\left|\Im z_{n}\right| \leq \theta\right\}, \quad T>0, \theta \geq 0 .
$$

Set $E_{0}=F \times\left\{z_{n}: \Re z_{n}=0,\left|\Im z_{n}\right| \leq \theta\right\}$, and let $\varphi\left(z^{\prime}, z_{n}\right) \in \mathcal{O}\left(E_{0}\right)$. Then there is a small $\delta>0$ such that $\varphi\left(z^{\prime}, z_{n}\right)$ is defined on

$$
F \times\left\{z_{n}:\left|\Re z_{n}\right| \leq \delta,\left|\Im z_{n}\right| \leq \theta+\delta\right\}
$$

Set

$$
\begin{aligned}
\ell & =\left\{z_{n}: \Re z_{n}=0,-\theta-\delta \leq \Im z_{n} \leq \theta+\delta\right\}, \\
E_{1} & =F \times\left\{z_{n}:-T \leq \Re z_{n} \leq \delta,\left|\Im z_{n}\right| \leq \theta\right\}, \\
E_{2} & =F \times\left\{z_{n}:-\delta \leq \Re z_{n} \leq T,\left|\Im z_{n}\right| \leq \theta\right\},
\end{aligned}
$$

where $\ell$ is positively oriented as $\Im z_{n}$ increases. We define Cousin's integral of $\varphi\left(z^{\prime}, z_{n}\right)$ along $\ell$ by

$$
\Phi\left(z^{\prime}, z_{n}\right)=\frac{1}{2 \pi i} \int_{\ell} \frac{\varphi\left(z^{\prime}, \zeta_{n}\right)}{\zeta_{n}-z_{n}} d \zeta_{n}
$$

Then $\Phi\left(z^{\prime}, z_{n}\right)$ is holomorphic on $\left(E_{1} \cup E_{2}\right) \backslash(F \times \ell)$. After analytic continuations we obtain $\Phi_{j}\left(z^{\prime}, z_{n}\right) \in$ $\mathcal{O}\left(E_{j}\right)(j=1,2)$ satisfying

$$
\Phi_{1}\left(z^{\prime}, z_{n}\right)-\Phi_{2}\left(z^{\prime}, z_{n}\right)=\varphi\left(z^{\prime}, z_{n}\right), \quad\left(z^{\prime}, z_{n}\right) \in E_{1} \cap E_{2} .
$$

We call this the Cousin decomposition of $\varphi\left(z^{\prime}, z_{n}\right)$.

The rest is the same as in the proof of [17] Lemma 4.3.7.

By the Weak Coherence Theorem 1.2 and Lemma 3.4 we have:

Theorem 3.9 (Syzygy for $\mathscr{I}\langle S\rangle$ ). Let $S$ be a complex submanifold of a neighborhood of a closed cuboid $E\left(\Subset \mathbf{C}^{n}\right)$.

(i) $\mathscr{I}\langle S\rangle$ has a finite generator system on $E$.

(ii) Let $\left\{\sigma_{j}\right\}_{1 \leq j \leq N}$ be a finite generator system of $\mathscr{I}\langle S\rangle$ on $E$ with $\sigma_{j} \in \mathcal{O}(E)$. Then for every $\underline{\sigma} \in \Gamma(E, \mathscr{I}\langle S\rangle)(\sigma \in \mathcal{O}(E))$ there are holomorphic functions $a_{j} \in \mathcal{O}(E), 1 \leq j \leq N$, such that

$$
\sigma=\sum_{j=1}^{N} a_{j} \cdot \sigma_{j} \quad(\text { on } E) .
$$

\subsection{Oka's Jôku-Ikô}

Let $\mathrm{P}$ be an open cuboid in $\mathbf{C}^{n}$, and let $S \subset \mathrm{P}$ be a complex submanifold. The following is fundamental in the Oka theory.

Lemma 3.11 (Oka's Jôku-Ikô). Let $E \Subset \mathrm{P}$ be a closed cuboid. Then for every holomorphic function $g$ on $E \cap S(\Subset S)$ there exists an element $G \in \mathcal{O}(E)$ satisfying

$$
\left.G\right|_{E \cap S}=\left.g\right|_{E \cap S}
$$

\footnotetext{
${ }^{7)}$ With this writing we mean that $g$ is a holomorphic function in a neighborhood $V$ of $E \cap S$ in $S$. The notation will be
} used in sequel. 
Here, the equality holds in a neighborhood of $E \cap S$ in $S{ }^{8)}$

Remark. We call $G$ above a solution on $E$.

Proof. Notice that in the case of $E \cap S=\emptyset, G$ can be any holomorphic function on $E$, and the statement is true. We use induction on $\operatorname{dim} E$.

(a) Case of $\operatorname{dim} E=0$ : Since $E$ consists of one point, the assertion is clear.

(b) Case of $\operatorname{dim} E=\nu(\nu \geq 1)$ with the induction hypothesis that the case of $\operatorname{dim} E=\nu-1$ is true: By Theorem 3.9 (i) there is a finite generator system $\left\{\underline{\sigma_{j}}\right\}_{j=1}^{N}$ of $\mathscr{I}\langle S\rangle$ on a neighborhood $W(\subset \mathrm{P})$ of $E$ with $\sigma_{j} \in \mathcal{O}(W)$.

We may assume that $E$ is taken as in $(3.6)$. We set

$$
E_{t}=\left\{z=\left(z^{\prime}, z_{n}\right) \in E: \Re z_{n}=t\right\}, \quad-T \leq t \leq T .
$$

Since $\operatorname{dim} E_{t}=\nu-1$, the induction hypothesis implies that there is a solution $G_{t} \in \mathcal{O}\left(E_{t}\right)$ satisfying $\left.G_{t}\right|_{S \cap E_{t}}=\left.g\right|_{S \cap E_{t}}$. By the Heine-Borel Theorem there is a finite partition

$$
\begin{aligned}
& -T=t_{0}<t_{1}<\cdots<t_{L}=T, \\
& E_{\alpha}:=\left\{z=\left(z^{\prime}, z_{n}\right) \in E: t_{\alpha-1} \leq \Re z_{n} \leq t_{\alpha}\right\}, \quad 1 \leq \alpha \leq L,
\end{aligned}
$$

such that there are solutions $G_{\alpha} \in \mathcal{O}\left(E_{\alpha}\right)$ satisfying

$$
\left.G_{\alpha}\right|_{S \cap E_{\alpha}}=\left.g\right|_{S \cap E_{\alpha}} .
$$

Therefore, $\underline{G_{\alpha+1}-G_{\alpha}} \in \Gamma\left(E_{\alpha} \cap E_{\alpha+1}, \mathscr{I}\langle S\rangle\right)$. It follows from Theorem 3.9 (ii) that there are $a_{\alpha j} \in$ $\mathcal{O}\left(E_{\alpha} \cap E_{\alpha+1}\right)(1 \leq j \leq N)$ satisfying

$$
G_{\alpha+1}-G_{\alpha}=\sum_{j=1}^{N} a_{\alpha j} \sigma_{j} \quad\left(\text { on } E_{\alpha} \cap E_{\alpha+1}\right) .
$$

By the Cousin decomposition 3.7 of $a_{\alpha j}$ we write

$$
a_{\alpha j}=b_{\alpha j}-b_{\alpha+1 j} \quad\left(\text { on } E_{\alpha} \cap E_{\alpha+1}\right), \quad b_{\alpha j} \in \mathcal{O}\left(E_{\alpha}\right), b_{\alpha+1 j} \in \mathcal{O}\left(E_{\alpha+1}\right) .
$$

Then,

$$
G_{\alpha}+\sum_{j=1}^{N} b_{\alpha j} \sigma_{j}=G_{\alpha+1}+\sum_{j=1}^{N} b_{\alpha+1 j} \sigma_{j} \quad\left(\text { on } E_{\alpha} \cap E_{\alpha+1}\right) .
$$

Thus this yields a solution $H_{\alpha+1}$ on $E_{\alpha} \cup E_{\alpha+1}$; for this procedure we say that we merge the solutions $G_{\alpha}$ and $G_{\alpha+1}$ to obtain a solution $H_{\alpha+1}$ on $E_{\alpha} \cup E_{\alpha+1}$.

Starting from $\alpha=1$, we merge $G_{1}$ and $G_{2}$ to obtain a solution $H_{2}$ on $E_{1} \cup E_{2}$. We then merge $H_{2}$ and $G_{3}$ to obtain a solution $H_{3}$ on $E_{1} \cup E_{2} \cup E_{3}$. Repeating this procedure up to $\alpha=L-1$, we obtain a solution $H_{L}$ on $E=\bigcup_{\alpha=1}^{L} E_{\alpha}$, and set $G=H_{L}$ : This finishes the proof of Lemma 3.11

8) The formulation of this lemma and the proof below should be new. 
Remark 3.17. We call the above induction argument cuboid induction on dimension, which will be used furthermore in the sequel.

It is well-known that Oka's Jôku-Ikô Lemma 3.11 immediately implies (cf., e.g., [17] Lemma 4.4.17):

Theorem 3.18 (Runge-Weil-Oka Approximation). Let $\Delta \Subset \Omega$ be an analytic polyhedron of a domain

$\Omega\left(\subset \mathbf{C}^{n}\right)$. Then every holomorphic function on the closure $\bar{\Delta}$ is uniformly approximated on $\bar{\Delta}$ by elements of $\mathcal{O}(\Omega)$.

\section{Cousin I/II, ̄̄, Extension and Levi's (Hartogs' Inverse) Prob- lems}

The aim of this section is to show how the result obtained in the previous section is applied to solve the titled problems.

\subsection{Cousin I/II, and $\bar{\partial}$-Equation}

We will give one unified proof to all of the three problems. We recall them: Let $\Omega \subset \mathbf{C}^{n}$ be a domain, let $\Omega=\bigcup_{\alpha \in \Lambda} U_{\alpha}$ be an open covering, and let $\mathscr{M}\left(U_{\alpha}\right)$ denote the set of all meromorphic functions in $U_{\alpha}$.

I (Cousin I) For given $f_{\alpha} \in \mathscr{M}\left(U_{\alpha}\right)(\alpha \in \Lambda)$ satisfying $f_{\alpha}-f_{\beta} \in \mathcal{O}\left(U_{\alpha} \cap U_{\beta}\right)$ (Cousin-I data), find $F \in \mathscr{M}(\Omega)$ (called a solution) with $\left.F\right|_{U_{\alpha}}-f_{\alpha} \in \mathcal{O}\left(U_{\alpha}\right)$ for all $\alpha \in \Lambda$.

II (Cousin II, Oka Principle) Here we assume that $U_{\alpha}$ are simply-connected. Let $f_{\alpha} \in \mathscr{M}^{*}\left(U_{\alpha}\right)$ $(\alpha \in \Lambda)$ be locally non-zero meromorphic functions satisfying

(a) $f_{\alpha} / f_{\beta} \in \mathcal{O}^{*}\left(U_{\alpha} \cap U_{\beta}\right)$ (nowhere vanishing holomorphic functions) (Cousin-II data),

(b) (Topological condition) there are nowhere vanishing continuous functions $\psi_{\alpha} \in \mathscr{C}^{*}\left(U_{\alpha}\right)$ with $\psi_{\alpha} / \psi_{\beta}=f_{\beta} / f_{\alpha}$ on $U_{\alpha} \cap U_{\beta}$.

Find $F \in \mathscr{M}^{*}(\Omega)$ with $\left.F\right|_{U_{\alpha}} / f_{\alpha} \in \mathcal{O}^{*}\left(U_{\alpha}\right)$ for all $\alpha \in \Lambda$.

We may take a continuous branch $h_{\alpha}:=\log \psi_{\alpha}$ in each $U_{\alpha}$. It follows that

$$
\left.h_{\alpha}\right|_{U_{\alpha} \cap U_{\beta}}-\left.h_{\beta}\right|_{U_{\alpha} \cap U_{\beta}} \in \mathcal{O}\left(U_{\alpha} \cap U_{\beta}\right) .
$$

The problem to find the solution $F$ above is reduced to the following problem:

(*) For a given family $\left\{h_{\alpha}\right\}$ of continuous functions satisfying (4.1), find a continuous function $H$ (called a solution) in $\Omega$ such that for every $U_{\alpha}$

$$
\left.H\right|_{U_{\alpha}}-h_{\alpha} \in \mathcal{O}\left(U_{\alpha}\right) \text {. }
$$

III ( $\bar{\partial}$-Equation) For a given $C^{\infty}$-(0,1)-form $u$ on $\Omega$ with $\bar{\partial} u=0$, find a $C^{\infty}$-function $g$ (called a solution) on $\Omega$ with $\bar{\partial} g=u$.

Locally, by Dolbeault's lemma, there is a solution $f$ of this problem in a neighborhood of a point of $\Omega$. Thus, there are an open covering $\left\{U_{\alpha}\right\}_{\alpha \in \Lambda}$ of $\Omega$ and $C^{\infty}$-functions $g_{\alpha}$ on $U_{\alpha}$ such that 
$\bar{\partial} g_{\alpha}=\left.u\right|_{U_{\alpha}}$. Then, the present problem is equivalent to find a $C^{\infty}$-function $G$ (solution) on $\Omega$ with $\left.G\right|_{U_{\alpha}}-g_{\alpha} \in \mathcal{O}\left(U_{\alpha}\right)$ for all $\alpha \in \Lambda$.

Convention. For a unified treatment for the above problems, we introduce an "argument $\chi$ " representing one of I-III above,: Problem- $\chi$ means one of Problems I-III above, where Problem-II means Problem $(\star)$, and a $\chi$-solution means a solution of the corresponding Problem- $\chi$.

Remark 4.2. If $\Psi$ is so obtained in Cousin-II Problem above, then $F_{1}=f_{\alpha} e^{\log \psi_{\alpha}-\Psi} \in \mathscr{M}^{*}(\Omega)$ satisfies the required property for $F$. Then we have a homotopy,

$$
F_{t}=f_{\alpha} e^{\log \psi_{\alpha}-t \Psi}, \quad 0 \leq t \leq 1,
$$

from the topologically assumed function $F_{0}\left(=f_{\alpha} \psi_{\alpha}\right)$ to an aimed analytic (meromorphic) function $F_{1}$.

Remark 4.3. The common property of Problem- $\chi$ that we will use is the following: If $f$ and $f^{\prime}$ are two solutions of Problem- $\chi$ on an open set $U$ in general, then $f-f^{\prime} \in \mathcal{O}(U)$.

We begin with:

Lemma 4.4. Let $\mathrm{P}$ be an open cuboid in $\mathbf{C}^{n}$ and let $S$ be a complex submanifold of $\mathrm{P}$. We consider Problem- $\chi$ defined on $S$. Let $E \Subset \mathrm{P}$ be a closed cuboid. Then there is a $\chi$-solution on $E \cap S(\Subset S), 99$

Proof. We use cuboid induction on dimension.

(a) Case of $\operatorname{dim} E=0$ : It is clear by definition.

(b) Case of $\operatorname{dim} E=\nu(\nu \geq 1)$ with the induction hypothesis that the case of $\operatorname{dim} E=\nu-1$ holds: Without loss of generality we may assume that $E$ is given as in (3.6), and let $E_{t}$ be as in (3.12). Since $\operatorname{dim} E_{t}=\nu-1$, the induction hypothesis implies the existence of a $\chi$-solution $\Phi_{t}$ on $E_{t} \cap S(\Subset S)$. Then, by the Heine-Borel Theorem there are a partition of $[-T, T], E_{\alpha}(1 \leq \alpha \leq L)$ as in $(3.13)$, and $\chi$-solutions $\Phi_{\alpha}$ on $E_{\alpha} \cap S(\Subset S)$.

If $E_{\alpha} \cap E_{\alpha+1} \cap S \neq \emptyset$, we say that $E_{\alpha}$ and $E_{\alpha+1}$ is pairwise connected on $S$. It is sufficient to prove the existence of a $\chi$-solution for each maximal sequence of $E_{\alpha}$ pairwise connected on $S$,

$$
E_{\alpha_{0}} \cup E_{\alpha_{0}+1} \cup \cdots \cup E_{\alpha_{1}} \text {. }
$$

For simplicity we suppose that $\alpha_{0}=1$. It follows from Remark 4.3 that for $1 \leq \alpha \leq \alpha_{1}$

$$
\Phi_{\alpha+1}-\Phi_{\alpha} \in \Gamma\left(E_{\alpha} \cap E_{\alpha+1} \cap S, \mathcal{O}_{S}\right) .
$$

By Oka's Jôku-Ikô Lemma 3.11, there is a holomorphic function $H_{\alpha} \in \mathcal{O}\left(E_{\alpha} \cap E_{\alpha+1}\right)$ such that

$$
\left.H_{\alpha}\right|_{E_{\alpha} \cap E_{\alpha+1} \cap S}=\Phi_{\alpha+1}-\Phi_{\alpha} .
$$

By the Cousin decomposition of $H_{\alpha}$ as in (3.7) we have $\tilde{H}_{\alpha} \in \mathcal{O}\left(E_{\alpha}\right)$ and $\tilde{H}_{\alpha+1} \in \mathcal{O}\left(E_{\alpha+1}\right)$ such that

$$
H_{\alpha}=\tilde{H}_{\alpha}-\tilde{H}_{\alpha+1} \quad\left(\text { on } E_{\alpha} \cap E_{\alpha+1}\right) .
$$

\footnotetext{
9) Cf. footnote 7) at p. 8
} 
We infer from 4.8 and 4.16 that

$$
\Phi_{\alpha}+\left.\tilde{H}_{\alpha}\right|_{E_{\alpha} \cap S}=\Phi_{\alpha+1}+\left.\tilde{H}_{\alpha+1}\right|_{E_{\alpha+1} \cap S} \quad \text { on } E_{\alpha} \cap E_{\alpha+1} \cap S(\Subset S) .
$$

Note that $\Phi_{\alpha}+\left.\tilde{H}_{\alpha}\right|_{E_{\alpha} \cap S}\left(\operatorname{resp} . \Phi_{\alpha+1}+\left.\tilde{H}_{\alpha+1}\right|_{E_{\alpha+1} \cap S}\right)$ is a $\chi$-solution on $E_{\alpha} \cap S(\Subset S)\left(\right.$ resp. $\left.E_{\alpha+1} \cap S(\Subset S)\right)$. Thus, from 4.9 we obtain a merged $\chi$-solution $\Psi_{\alpha+1}$ on $\left(E_{\alpha} \cup E_{\alpha+1}\right) \cap S(\Subset S)$ from $\Phi_{\alpha}$ and $\Phi_{\alpha+1}$.

Now, from $\Phi_{1}$ and $\Phi_{2}$ we obtain a merged $\chi$-solution $\Psi_{2}$ on $\left(E_{1} \cup E_{2}\right) \cap S(\Subset S)$. We then obtain a merged $\chi$-solution $\Psi_{3}$ on $\left(E_{1} \cup E_{2} \cup E_{3}\right) \cap S(\Subset S)$ from $\Psi_{2}$ and $\Phi_{3}$, and so on; we obtain a $\chi$-solution on $\left(\bigcup_{\alpha=1}^{\alpha_{1}} E_{\alpha}\right) \cap S(\Subset S)$.

Definition 4.10. A complex manifold $M$ is said to be holomorphically convex if for every compact subset $K \Subset M$ the holomorphically convex hull of $K$ defined by

$$
\hat{K}_{M}=\{a \in M:|f(a)| \leq \max \{|f(x)|: x \in K\}
$$

is again compact.

Theorem 4.11. Let $\Omega$ be a holomorphically convex domain. Then Problem- $\chi$ on $\Omega$ has a $\chi$-solution on $\Omega$.

Proof. We take an increasing sequence of analytic polyhedra of $\Omega$,

$$
\Delta_{1} \Subset \Delta_{2} \Subset \Delta_{3} \Subset \cdots, \quad \bigcup_{\nu=1}^{\infty} \Delta_{\nu}=\Omega
$$

For each $\nu$ we let $\phi_{\nu}: \bar{\Delta}_{\nu} \rightarrow \overline{\mathrm{P}}_{\nu}$ be the Oka map (a holomorphic proper embedding) of $\bar{\Delta}_{\nu}$ into a closed polydisk $\overline{\mathrm{P}}_{\nu}$, which extends from a neighborhood $U_{\nu}$ of $\bar{\Delta}_{\nu}$ into a polydisk, biholomorphic to an open cuboid $\mathrm{P}_{\nu}\left(\ni \overline{\mathrm{P}}_{\nu}\right)$. Then, the image $\phi_{\nu}\left(U_{\nu}\right)$ is a complex submanifold of $\mathrm{P}_{\nu}$. We identify $U_{\nu}$ with the image $\phi_{\nu}\left(U_{\nu}\right)$.

By Lemma 4.4 there is a $\chi$-solution $G_{\nu}$ on every $\bar{\Delta}_{\nu}$. Put $F_{1}=G_{1}$ on $\bar{\Delta}_{1}$. Suppose that $\chi$-solutions $F_{\nu}$ on $\bar{\Delta}_{\nu}, 1 \leq \nu \leq \mu$, are determined so that

$$
\left\|F_{\nu+1}-F_{\nu}\right\|_{\bar{\Delta}_{\nu}}<\frac{1}{2^{\nu}}, \quad 1 \leq \nu \leq \mu .
$$

Let $G_{\mu+1}$ be a $\chi$-solution on $\bar{\Delta}_{\mu+1}$. Since $\left.G_{\mu+1}\right|_{\bar{\Delta}_{\mu}}-F_{\mu} \in \mathcal{O}\left(\bar{\Delta}_{\mu}\right)$, by Theorem 3.18 there is an element $h_{\mu+1} \in \mathcal{O}\left(\bar{\Delta}_{\mu+1}\right)$ with

$$
\left\|\left.G_{\mu+1}\right|_{\bar{\Delta}_{\mu}}-F_{\mu}-h_{\mu+1}\right\|_{\bar{\Delta}_{\mu}}<\frac{1}{2^{\mu+1}} .
$$

Setting $F_{\mu+1}=G_{\mu+1}-h_{\mu+1}$, we see that (4.13) holds up to $\mu+1$. Inductively, we have $\chi$-solutions $F_{\nu}$ on $\bar{\Delta}_{\nu}$ satisfying 4.13 , and the series

$$
F=F_{\mu}+\sum_{\nu=\mu}^{\infty}\left(F_{\nu+1}-F_{\nu}\right)
$$

converges locally uniformly and the limit gives rise to a $\chi$-solution on $\Omega$.

Remark 4.14. As easily seen, the above proof of Theorem 4.11 works on Stein manifolds, which is defined by: 
Definition 4.15. A complex manifold $M$ is said to be Stein if the following conditions are satisfied.

(i) $M$ satisfies the second countability axiom:

(ii) (Holomorphic separation) For every two distinct two point $a, b \in M$ there is a holomorphic function $f \in \mathcal{O}(M)$ on $M$ with $f(a) \neq f(b)$ :

(iii) For every point $a \in M$ there are holomorphic functions $f_{j} \in \mathcal{O}(M), 1 \leq j \leq n=\operatorname{dim}_{a} M$, such that $d f_{1}(a) \wedge \cdots \wedge d f_{n}(a) \neq 0:$

(iv) $M$ is holomorphically convex (see Definition 4.10).

\subsection{Extension Problem}

By means of the Weak Coherence Theorem 1.2 we consider the extension problem (interpolation problem) from a complex submanifold in a holomorphically convex domain.

Theorem 4.16. Let $\Omega \subset \mathbf{C}^{n}$ be a holomorphically convex domain and let $S \subset \Omega$ be a complex submanifold. Then the restriction map

$$
\left.F \in \mathcal{O}(\Omega) \rightarrow F\right|_{S} \in \mathcal{O}(S)
$$

is a surjection.

Proof. We take analytic polyhedra $\Delta_{\nu} \Subset \Omega$ and Oka maps $\phi_{\nu}: \bar{\Delta}_{\nu}\left(\Subset U_{\nu}\right) \rightarrow \overline{\mathrm{P}}_{\nu}\left(\Subset \mathrm{P}_{\nu}\right)(\nu=1,2, \ldots)$ as in the proof of Theorem 4.11. By Theorem 3.9 (i) there is a finite generator system $\left\{\underline{\sigma}_{\nu j}\right\}_{j=1}^{N_{\nu}}$ of $\mathscr{I}\left\langle S \cap \mathrm{P}_{\nu}\right\rangle$ on each $\overline{\mathrm{P}}_{\nu}\left(\Subset \mathrm{P}_{\nu}\right)$, where $U_{\nu}$ is identified with $\phi_{\nu}\left(U_{\nu}\right)$.

Let $f \in \mathcal{O}(S)$ be any element. By Oka's Jôku-Ikô Lemma 3.11 there are $G_{\nu} \in \mathcal{O}\left(\overline{\mathrm{P}}_{\nu}\right)$ with $\left.G_{\nu}\right|_{\bar{\Delta}_{\nu} \cap S}=$ $\left.f\right|_{\bar{\Delta}_{\nu} \cap S}(\nu=1,2, \ldots)$.

We set $F_{1}=\left.G_{1}\right|_{\bar{\Delta}_{1}}$. Suppose that $F_{\nu} \in \mathcal{O}\left(\bar{\Delta}_{\nu}\right), 1 \leq \nu \leq \mu$, are determined so that

$$
F_{\nu}=\left.f\right|_{\bar{\Delta}_{\nu} \cap S}, \quad\left\|F_{\nu+1}-F_{\nu}\right\|_{\overline{\mathrm{P}}_{\nu}}<\frac{1}{2^{\nu}}, \quad 1 \leq \nu \leq \mu-1 .
$$

For $\nu=\mu+1$ we first note that $\left.\left(\left.G_{\mu+1}\right|_{\bar{\Delta}_{\mu}}-F_{\mu}\right)\right|_{\bar{\Delta}_{\mu} \cap S}=0$. By Lemma 3.11 there is an element $H_{\mu} \in \mathcal{O}\left({\overline{\mathrm{P}} \bar{\Delta}_{\mu}}\right)$ with $\left.H_{\mu}\right|_{\bar{\Delta}_{\mu}}=\left.G_{\mu+1}\right|_{\bar{\Delta}_{\mu}}-F_{\mu}$. Since $\underline{H}_{\mu} \in \Gamma\left(\overline{\mathrm{P}}_{\nu}, \mathscr{I}\langle S\rangle\right)$, by Theorem 3.9 (ii) there are $h_{\mu j} \in \mathcal{O}\left(\overline{\mathrm{P}}_{\mu}\right), 1 \leq j \leq N_{\mu+1}$, such that

$$
H_{\mu}=\sum_{j=1}^{N_{\mu+1}} h_{\mu j} \cdot \sigma_{\mu+1 j} \quad \text { on } \overline{\mathrm{P}}_{\mu} .
$$

Restricting this to $\bar{\Delta}_{\nu}$, we have

$$
\left.G_{\mu+1}\right|_{\bar{\Delta}_{\mu}}=F_{\mu}+\left.\sum_{j=1}^{N_{\mu+1}} h_{\mu j} \cdot \sigma_{\mu+1 j}\right|_{\bar{\Delta}_{\mu}} .
$$

Approximating $h_{\mu j}$ sufficiently close by $\tilde{h}_{\mu j} \in \mathcal{O}(\Omega)$ on $\bar{\Delta}_{\mu}$ (Theorem 3.18, and setting

$$
F_{\mu+1}=G_{\mu+1}-\sum_{j=1}^{N_{\mu+1}} \tilde{h}_{\mu j} \cdot \sigma_{\mu+1 j} \in \mathcal{O}\left(\bar{\Delta}_{\mu+1}\right)
$$


we have

$$
\left.F_{\mu+1}\right|_{\bar{\Delta}_{\mu+1} \cap S}=\left.f\right|_{\bar{\Delta}_{\mu+1} \cap S}, \quad\left\|F_{\mu+1}-F_{\mu}\right\|_{\bar{\Delta}_{\mu}}<\frac{1}{2^{\mu}} .
$$

Then the series

$$
F=F_{\mu}+\sum_{\nu=\mu}^{\infty}\left(F_{\nu+1}-F_{\nu}\right)
$$

converges locally uniformly to the limit $F \in \mathcal{O}(\Omega)$ with $\left.F\right|_{S}=f$.

Remark 4.18. The above proof of Theorem 4.16 works on Stein manifolds.

\subsection{Levi's (Hartogs' Inverse) Problem}

\subsubsection{Levi's (Hartogs' Inverse) Problem}

We first recall some basic terminologies (see, e.g., [9], [12, [17 for more details). Let $M$ be a connected complex manifold.

An upper semi-continuous function $\psi$ on $M$ is said to be plurisubharmonic if every restriction of $\psi$ to a 1-dimensional complex submanifold of any holomorphic local chart of $M$ is subharmonic. If $\psi$ is $C^{2}$-class, it is plurisubharmonic if and only if the hermitian matrix

$$
\left(\frac{\partial^{2} \psi}{\partial z_{j} \bar{\partial} z_{k}}\right) \geq 0 \quad \text { (semi-posititive definite) }
$$

where $z=\left(z_{j}\right)$ is a holomorphic local coordinate system of $M$; moreover, if

$$
\left(\frac{\partial^{2} \psi}{\partial z_{j} \bar{\partial} z_{k}}\right) \gg 0 \quad \text { (posititive definite) }
$$

then $\psi$ is said to be strongly plurisubharmonic.

If $M$ carries a continuous plurisubharmonic exhaustion $\psi(10)$, then $M$ is said to be pseudoconvex. Note that there are several equivalent definitions of being "pseudoconvex" (cf. [10, [9], 12, [14, [17, etc.).

A relatively compact domain $\Omega \Subset M$ is said to be strongly pseudoconvex if for every boundary point $b \in \partial \Omega$ there are a neighborhood $U$ of $b$ in $M$ and a strongly plurisubharmonic function $\phi$ on $U$ satisfying

$$
\Omega \cap U=\{x \in U: \phi(x)<0\} .
$$

It is known that a strongly pseudoconvex domain is pseudoconvex.

If $M$ is connected and there is a locally finite holomorphic map $\pi: M \rightarrow \mathbf{C}^{n}$ with $\operatorname{dim} M=n$, then we call $M$ a Riemann domain, in general; furthermore, if $\pi$ is locally (resp. non-) biholomorphic, we call $M$ a (resp. ramified) unramified Riemann domain; in this case, $M$ carries a Riemannian metric induced from the euclidean one on $\mathbf{C}^{n}$ through $\pi$, so that $M$ satisfies the second countability axiom.

It had been known that a Stein manifold is pseudoconvex: Levi's (Hartogs' Inverse) Problem had asked originally the converse for univalent domains of $\mathbf{C}^{n}$. K. Oka extended the problem for Riemann domains. (There was a necessity to do so (cf., e.g., [17 §5.1).)

\footnotetext{
${ }^{10)}$ A functions $\psi$ is called an exhaustion if $\{x \in M: \psi(x)<c\} \Subset M$ for all $c \in \mathbf{R}$.
} 


\subsubsection{Oka's method}

Notice that Oka's Jôku-Ikô Lemma 3.11 is sufficient to deduce Oka's Heftungslemma which, together with a method of an integral equation and the construction of a plurisubharmonic exhaustion on a pseudoconvex unramified Riemann domain, implies Levi's (Hartogs' Inverse) Problem (cf. Oka 25], 26], [31, [30], Andreotti-Narasimhan [1, Nishino [14]):

Theorem 4.19 (Oka, 1941/42/43/53; cf. \$5). Let $M$ be a unramified Riemann domain. If $M$ is pseudoconvex, then $M$ is Stein.

\subsubsection{Grauert's method}

In $1958 \mathrm{H}$. Grauert [8] gave another proof of Theorem 4.19 by proving the finite dimensionality of the first cohomology of coherent sheaves which was inspired by the Cartan-Serre Theorem for coherent sheaves on compact analytic spaces 11 ) We shall observe that the Weak Coherence Theorem 1.2 suffices for Grauert's method to prove Theorem 4.19 .

We use the first Čech cohomology $H^{1}(*, \mathcal{O})$. The following immediately follows from Theorem 4.11

Lemma 4.20. (i) If $\Omega$ be a holomorphically convex domain of $\mathbf{C}^{n}$, then $H^{1}(\Omega, \mathcal{O})=0$.

(ii) Let $M$ be a complex manifold with the second countability axiom, and let $\mathscr{U}=\left\{U_{\nu}\right\}$ be a Stein covering of $M$ (i.e., every $U_{\nu}$ is open and Stein). Then we have

$$
H^{1}(\mathscr{U}, \mathcal{O}) \cong H^{1}(M, \mathcal{O})
$$

Then we can apply Grauert's bumping method [8] to prove:

Theorem 4.21 (Grauert). Let $\Omega \Subset M$ be a relatively compact domain of a complex manifold $M$ with strongly pseudoconvex boundary. Then we have

$$
\operatorname{dim}_{\mathbf{C}} H^{1}(\Omega, \mathcal{O})<\infty
$$

Proof of Theorem 4.19 (i) The key of the proof is to show that a strongly pseudoconvex domain $\Omega \Subset M$ is Stein. Let $b \in \partial \Omega$ be a boundary point. By the definition of strong pseudoconvexity there are a neighborhood $U$ of $b$ in $M$ and a quadratic polynomial $P_{b}\left(z_{1}, \ldots, z_{n}\right)$ such that $U \cap \bar{\Omega} \cap\left\{P_{b}=0\right\}=\{b\}$. Then there is a little bit larger strongly pseudoconvex domain $\Omega_{\epsilon} \ni \Omega$ such that $\Omega_{\epsilon} \cap\left\{P_{b}=0\right\}$ is closed in $\Omega_{\epsilon}$. We consider Cousin-I data $\left(U_{0}, f_{\nu 0}\right)$ and $\left(U_{1}, f_{\nu 1}\right)$ of $\Omega_{\omega}$ such that for $\nu=1,2, \ldots$,

$$
\begin{array}{ll}
f_{\nu 0}=\frac{1}{P_{b}(z)^{\nu}} & \text { on } U_{0}=\Omega_{\epsilon} \cap U, \\
f_{\nu 1}=0 & \text { on } U_{1}=\Omega_{\epsilon} \backslash\left\{P_{b}=0\right\} .
\end{array}
$$

Theorem 4.21 applied to these Cousin-I data on $\Omega_{\epsilon}$ yields a meromorphic function $F_{b}$ on $\Omega_{\epsilon}$ such that $F_{b}$ is holomorphic in $U_{1}$, and in $U_{0}, F_{b}$ is written as

$$
F_{b}(z)=\frac{c_{\nu}}{P_{b}(z)^{\nu}}+\cdots+\frac{c_{1}}{P_{b}(z)}+\text { holomorphic term, }
$$

11) Cf. the footnote of 8 p. 466. The proof relies on L. Schwartz's finiteness theorem, whose rather simple, short and complete proof is found in [5] and [17] pp. 313-315. 
where $c_{\mu} \in \mathbf{C}(1 \leq \mu \leq \nu)$ and $c_{\nu} \neq 0$. Thus $\left.F_{b}\right|_{\Omega} \in \mathcal{O}(\Omega)$ and $\Omega$ being holomorphically convex is deduced.

(ii) To show the holomorphic separation of $\Omega$, we take two distinct points $Q_{1}, Q_{2} \in \Omega$. We may assume that $\pi\left(Q_{1}\right)=\pi\left(Q_{2}\right)=a \in \mathbf{C}^{n}$. Let $\phi(t), t \geq 0$, be any affine linear curve with $\phi(0)=a$. Then there is a unique lifting $\phi_{j}(t) \in \Omega, j=1,2$, such that $\phi_{j}(0)=Q_{j}$ and $\pi \circ \phi_{j}(t)=\phi(t)$. Since $\Omega$ is relatively compact, $\phi_{j}(t)$ hits the boundary $\partial \Omega$. We may assume that $\phi_{1}(t)$ hits $\partial \Omega$ first with $t=T \in \mathbf{R}$, so that $\phi_{j}([0, T]) \subset \bar{\Omega}(j=1,2)$ and $\phi_{1}(T) \in \partial \Omega$. Note that $\phi_{1}(T) \neq \phi_{2}(T)$. With setting $b=\phi_{1}(T)$ we have by 4.22 a meromorphic function $F_{b}$ in $\Omega_{\epsilon}$ which is holomorphic in $\Omega$.

We consider the Taylor expansions of $F_{b}$ at $Q_{1}$ and $Q_{2}$ in coordinates $\left(z_{1}, \ldots, z_{n}\right)$. Since $F_{b}$ has a pole at $\phi_{1}(T)$ and no pole at $\phi_{2}(T)$, those two expansions must be different. Therefore, there is some partial differential operator $\partial^{\alpha}=\partial^{|\alpha|} / \partial z_{1}^{\alpha_{1}} \cdots \partial z_{n}^{\alpha_{n}}$ with a multi-index $\alpha$ such that

$$
\partial^{\alpha} F_{b}\left(Q_{1}\right) \neq \partial^{\alpha} F_{b}\left(Q_{2}\right) \text {. }
$$

Since $\partial^{\alpha} F_{b}$ is holomorphic in $\Omega$, this finishes the proof.

Remark 4.23. (i) The idea of the proof above is inspired by Oka's unpublished paper in 1943 ([32]). Note that it is subtle how to deal with the holomorphic separation of an unramified Riemann domain. In Hörmander [12] the holomorphic separation is included in the definition of Riemann domains. In 17. Chap. 7 we used Grauert's Theorem 4.21 for a non-singular geometric ideal sheaves, which can be also deduced from our Weak Coherence Theorem, but then it involves a sheaf-cohomological argument. In Gunning-Rossi [10] and in Nishino [14] they gave their own proofs.

(ii) The proof presented above provides a complete proof of Levi's (Hartogs' Inverse) Problem without the sheaf cohomology theory nor $L^{2}-\bar{\partial}$ method.

\section{$5 \quad$ Historical remarks}

Here, cf. [17] Chap. 9 "On Coherence", and cf., e.g., Lieb [13] for the general background.

Oka's Theorem 4.19 was first proved for univalent domains $\Omega \subset \mathbf{C}^{2}$ by Oka [25] (announcement) in 1941, and the full paper [26] was published in 1942 with a comment of the validity for $n \geq 3$.

In 1943 Oka proved Theorem 4.19 for unramified Riemann domains of general dimension $\geq 2$ in a series of research reports of pp. 109 in total, sent to Teiji Takagi: The reports were written in Japanese and unpublished (see [31, [32]). He remarked this fact three times, first in his survey note [27] (1949), VIII 29] (1951) and IX 30] (1953). The paper 27] has not been referred very much, but it should have a significant interest, for it was written during the submission of VII [28] before the publication; he had sent it to H. Cartan 1948 one year ago (through the hands of S. Kakutani and A. Weil), and in return he had received Cartan's conjectural (or experimental) paper [3] referred in [27. He surveyed the state of the development of analytic function theory of several variables at the time.

In the 1943 reports to Takagi he did not use Weierstrass' Preparation Theorem, but he was writing a primitive form of the notion of coherence and non-reduced structures of analytic subsets; the study later led to the notion "idéaux de domaines indéterminés", coherence in 1948 ([28]). The key of Oka's 
proof of Theorem 4.19 was his "Heftungslemma". In [25] and 26] he proved Heftungslemma by Weil's integral, but in 1943 (31 no. 1, 32]) he replaced Weil's integral by simple Cauchy's integral, proving "Oka's Jôku-Ikô" for unramified Riemann domains.

In 1949 S. Hitotsumatsu [11] written in Japanese gave a proof of Oka's Heftungslemma by Weil's integral to solve Levi's (Hartogs' Inverse) Problem in general dimension $n \geq 2$; here he gave no argument of plurisubharmonic exhaustions on pseudoconvex unramified Riemann domains, and so the result might hold only for univalent domains.

In 1953 Oka 30 proved Theorem 4.19 above by making use of his First and Second Coherence Theorems obtained in [28]: the Third Coherence Theorem was not used there.

In 1954 Bremermann [2] and Norguet [19] independently proved Theorem 4.19 for univalent domains $\Omega \subset \mathbf{C}^{n}$ with general $n \geq 2$, generalizing Oka's Heftungslemma by means of Weil's integral, similarly to Hitotsumatsu [11.

Concluding Remark (Oka's Problem). It is interesting to learn that Oka invented and proved three fundamental coherence theorems by means of Weierstrass' Preparation Theorem in order to treat the pseudoconvexity problem on singular ramified Riemann domains. Levi's (Hartogs' Inverse) Problem for ramified Riemann domains has a counter-example (Fornæss [7]), but in the same time there is a positive case for which Levi's (Hartogs' Inverse) Problem is affirmative ([18]). Because of the above historical facts we may call the following question

Oka's Problem: What is necessary and/or sufficient for the validity of Levi's (Hartogs' Inverse) Problem on a ramified Riemann domain $X\left(\right.$ over $\left.\mathbf{C}^{n}\right)$ ? :

This is open even when $X$ is non-singular.

\section{References}

[1] A. Andreotti and R. Narasimhan, Oka's Heftungslemma and the Levi problem for complex spaces, Trans. Amer. Math. Soc. 111 (1964), 345-366.

[2] H.J. Bremermann, Über die Äquivalenz der pseudokonvexen Gebiete und der Holomorphiegebiete im Raum von $n$ komplexen Veränderlichen, Math. Ann. 128 (1954), 63-91.

[3] H. Cartan, Idéaux de fonctions analytiques de $n$ variables complexes, Ann. Sci. École Norm. Sup. 61 (1944), $149-197$.

[4] H. Cartan, Idéaux et modules de fonctions analytiques de variables complexes Bull. Soc. Math. France $\mathbf{7 8}$ (1950), 29-64.

[5] J.-P. Demailly, Complex Analytic and Differentiable Geometry, 2012, www-fourier.ujfgrenoble.fr/ demailly/ .

[6] J. Dieudonné, Abrégé d'histoire des mathématiques : 1700-1900, I, Hermann, Paris, 1986.

[7] J.E. Fornæss, A counterexample for the Levi problem for branched Riemann domains over $\mathbf{C}^{n}$, Math. Ann. 234 (1978), 275-277.

[8] H. Grauert, On Levi's problem and the imbedding of real-analytic manifolds, Ann. Math. 68 (1958), 460-472.

[9] H. Grauert and R. Remmert, Theorie der Steinschen Räume, Grundl. Math. Wiss. 227 Springer-Verlag, Berlin, 1977: Translated to English by A. Huckleberry, Theory of Stein Spaces, Springer-Verlag, Berlin, 1979: Translated to Japanese by K. Miyajima, Stein Kukan Ron, Springer, Tokyo, 2009D

[10] R.C. Gunning and H. Rossi, Analytic Functions of Several Complex Variables, Prentice-Hall, 1965.

[11] S. Hitotsumatsu, On Oka's Heftungs Theorem (Japanese), Sugaku 1 (4) (1949), 304-307, Math. Soc. Jpn.

[12] L. Hörmander, Introduction to Complex Analysis in Several Variables, Third Edition, North-Holland, 1989.

[13] I. Lieb, Le problème de Levi, Gaz. Math. Soc. Math. Fr. 115 (2008), 9-34.

[14] T. Nishino, Function Theory in Several Complex Variables (in Japanese), The University of Tokyo Press, Tokyo, 1996; Translation into English by N. Levenberg and H. Yamaguchi, Amer. Math. Soc. Providence, R.I., 2001. 
[15] J. Noguchi, Another direct proof of Oka's Theorem (Oka IX), J. Math. Sci. Univ. Tokyo 19 (2012), 1-15.

[16] Noguchi, J., A remark to a division algorithm in the proof of Oka's First Coherence Theorem, Internat. J. Math. 26 No. 4 (2015), DOI: 10.1142/S0129167X15400054, 8 pp.

[17] J. Noguchi, Analytic Function Theory of Several Variables -Elements of Oka's Coherence, Springer, Singapore, 2016; translated from Analytic Function Theory of Several Variables (in Japanese), Asakura-Shoten, Tokyo, 2013.

[18] J. Noguchi, Inverse of Abelian integrals and ramified Riemann domains, Math. Ann. 367 No. 1 (2017), 229-249.

[19] F. Norguet, Sur les domains d'holomorphie des fonctions uniformes de plusieurs variables complexes (Passage du local au global), Bull. Soc. Math. France 82 (1954), 137-159.

[20] K. Oka, Sur les fonctions analytiques de plusieurs variables- I- Domaines convexes par rapport aux fonctions rationnelles, J. Sci. Hiroshima Univ. Ser. A 6 (1936), 245-255.

[21] K. Oka, Sur les fonctions analytiques de plusieurs variables- II - Domaines d'holomorphie, J. Sci. Hiroshima Univ. Ser. A 7 (1937), 115-130.

[22] K. Oka, Sur les fonctions analytiques de plusieurs variables- III- Deuxième problème de Cousin, J. Sci. Hiroshima Univ. 9 (1939), 7-19.

[23] K. Oka, Sur les fonctions analytiques de plusieurs variables- IV - Domaines d'holomorphie et domaines rationnellement convexes, Jpn. J. Math. 17 (1941), 517-521.

[24] K. Oka, Sur les fonctions analytiques de plusieurs variables- V-L'intégrale de Cauchy, Jpn. J. Math. 17 (1941), 523-531.

[25] K. Oka, Sur les domaines pseudoconvexes, Proc. Imperial Acad. Tokyo 17 (1941), 7-10.

[26] K. Oka, Sur les fonctions analytiques de plusieurs variables - VI Domaines pseudoconvexes, Tôhoku Math. J. 49 (1942), 15-52.

[27] K. Oka, Note sur les fonctions analytiques de plusieurs variables, Kōdai Math. Sem. Rep. (1949) no. 5-6, $15-18$.

[28] K. Oka, Sur les fonctions analytiques de plusieurs variables - VII Sur quelques notions arithmétiques, Bull. Soc. Math. France 78 (1950), 1-27

[29] K. Oka, Sur les fonctions analytiques de plusieurs variables - VIII Lemme fondamental, J. Math. Soc. Jpn. 3 (1951) No. 1, 204-214, No. 2, 259-278.

[30] K. Oka, Sur les fonctions analytiques de plusieurs variables - IX Domaines finis sans point critique intérieur, Jpn. J. Math. 23 (1953), 97-155.

[31] K. Oka, Posthumous Papers of Kiyoshi Oka, Eds. T. Nishino and A. Takeuchi, Kyoto, 1980-1983: Oka Kiyoshi Collection, Library of Nara Women's University, URL 'http://www.lib.nara-wu.ac.jp/oka/'.

[32] K. Oka, On analytic functions of several variables: XI - Pseudoconvex domains and finite domains of holomorphy, Some theorems on finite domains of holomorphy, 1943: URL http://www.ms.utokyo.ac.jp/ noguchi/oka/", (A1).

Graduate School of Mathematical Sciences University of Tokyo (Emeritus) Komaba, Meguro-ku, Tokyo 153-8914 Japan e-mail: noguchi@ms.u-tokyo.ac.jp

(Revised 3/July/2018) 